\title{
Spiral selection as a free boundary problem
}

\author{
David A. Kessler ${ }^{\mathrm{a}}$ and Herbert Levine ${ }^{\mathrm{b}}$ \\ a Department of Physics, Unicersity of Michigan, Ann Arbor, MI 48109, USA \\ 'Department of Physics and Institute for Nonlinear Science, Unilersity of California at San Diego, La Jolla, CA 92093, USA
}

\begin{abstract}
We present a new formulation of the spiral selection problem for the Belousov-Zhabotinsky reaction. In particular, we focus on deriving an exact integro-differential shape equation and discuss the possible behavior of solutions to this equation. We also present some new results on the asymptotic (far from core) structure of spirals in the Fife scaling regime.
\end{abstract}

\section{Introduction}

One of the most striking examples of spatial pattern formation in non-equilibrium systems is the rotating spiral seen in diffusion-coupled excitable media ${ }^{\# 1}$. The most studied example to date is the Belousov-Zhabotinsky (BZ) case [2-5], but similar structures appear in systems as diverse as catalysis on platinum surfaces [6] and aggregation of slime mold amoeba [7]. Remarkably and regrettably there is as yet no first principles calculation of a spiral shape in any reaction-diffusion system.

This work attempts to formulate and treat spiral patterns in a simplified Oregonator model ${ }^{\# 2}$ to be described shortly. Some of the ideas have appeared elsewhere in preliminary form, but here we offer some new results and a new perspective on this problem. The basic ingredient of our method is the rewriting of the spiral pattern problem as a free boundary problem for the thin reaction zone of the fast reaction. This approach was advocated long ago by Fife, but will be reformulated here in a novel manner.

The outline of this paper is as follows. First, we briefly review the Fife regime of the Oregonator model and explain why this scaling is crucial for the spiral shape determination. Next, we derive asymptotic corrections (far from the core) to the spiral shape and discuss the utility of these results. Finally, we derive an integro-differential shape equation in which the rotation frequency appears as an eigenvalue. We present a general argument as to why the spectrum of frequencies might be discrete, corresponding to the experimental observation of unique spiral structures at fixed conditions. This selection occurs despite the fact that the asymptotic expansion allows for a continuous frequency. Finally, we summarize our findings and offer some conjectures regarding spiral tip meandering.

\section{Fife scaling}

Let us briefly review the Oregonator model with the asymptotic wave velocity in the Fife region [9]. What this means is the following: the Oregonator equations have the form

$$
\begin{aligned}
& \dot{u}=\epsilon \nabla^{2} u+\frac{f(u, v)}{\epsilon}, \\
& \dot{i}=\epsilon \nabla^{2} u+g(u, v),
\end{aligned}
$$

\footnotetext{
\# For a general introduction to excitable media, see ref. [1].

\#2 For a discussion of the Oregonator model, see ref. [8].
} 
where the equation $f(u, v)=0$ admits multiple stable solutions $u=u_{+}(v)$ for $v$ in some range. The ratio of reaction rates, $\epsilon$, is small and hence as reviewed by Keener and Tyson [10], one can assume that $u=u_{+}(v)$ or $u_{-}(v)$ except for thin reaction zone. Actually, this methodology crucially depends on the assumption that the relevant length scales in the spiral pattern are never comparable to the reaction zone width $\epsilon$; this will be important in the discussion below.

In the reaction zone, $v$ can be taken constant, and a domain wall solution which proceeds from $u_{+}$to $u_{-}$(or vice versa) constructed. At one particular value of $v$, the stall concentration $v_{\mathrm{s}}$, this domain wall is motionless. For $v \sim v_{\mathrm{s}}$, we can linearize the relationship between velocity and concentration; including curvature effects, this leads to

$$
\left(c_{\mathrm{n}}+\epsilon \gamma \kappa\right) \nu_{0}^{\prime}=\left(v-v_{\mathrm{s}}\right)
$$

for some constant $\nu_{0}^{\prime}, c_{\mathrm{n}}$ the normal velocity and $\kappa$ the curvature. The constant $\gamma$ should equal 1 for the model given in eqs. (1), (2) but we leave it as an arbitrary additional parameter, which can be related to a ratio of unequal diffusivities for the two chemical species.

The Fife limit $[9,11]$ arises from the assumption that we are dealing with a wave with $c \sim \epsilon^{1 / 3}$. From the above equation, uniformity demands that lengths scale as $\epsilon^{2 / 3}$, and $v-v_{\mathrm{s}} \sim \epsilon^{1 / 3}$. In the dynamical equation for $v$, the function $g$ can be evaluated at the stall value $v_{\mathrm{s}}$. Combining all of these comments leads to the free boundary problem $[9,11]$

$$
\nabla^{2} \tilde{v} \pm a_{ \pm}=\partial \tilde{v} / \partial t
$$

with $\tilde{v}, \tilde{v}^{\prime}$ continuous across zones and where we must satisfy the rescaled version of eq. (3):

$$
\left(\tilde{c}_{\mathrm{n}}+\gamma \tilde{\kappa}\right) \nu_{0}^{\prime}=\tilde{v}
$$

Here $a_{ \pm}= \pm g\left(u_{ \pm}\left(v_{\mathrm{s}}\right), v_{\mathrm{s}}\right)$.

To find a spiral solution of the above equations, we assume a uniformly rotating field and replace $\nabla^{2}-\partial / \partial t$ by

$$
\frac{\partial^{2}}{\partial r^{2}}+\frac{1}{r} \frac{\partial}{\partial r}+\frac{1}{r^{2}} \frac{\partial^{2}}{\partial \phi^{2}}+\omega \frac{\partial}{\partial \phi}
$$

Also, $c_{\mathrm{n}}=\omega \hat{\boldsymbol{n}} \cdot \hat{\boldsymbol{\phi}}$. We must then find a curve $\Phi_{0}(r)$ for the reaction front such that the boundary condition (5) is satisfied by a continuous field $\tilde{v}$. There are two methods to do this; we can eliminate $\tilde{v}$ to find a shape equation for $\Phi_{0}$ or we can map the spiral to a simpler region and attempt to solve the resulting partial differential equation. These will be discussed in subsequent sections.

The most important thing to notice about the Fife limit is that $\epsilon$ has completely dropped out of the governing equations and boundary conditions. Let us therefore assume that there are indeed spiral solutions with the scaling assumed here $c \sim \epsilon^{1 / 3}$. Then, the physical scale of the spiral, being proportional to $\epsilon^{2 / 3}$, can be made arbitrarily large compared to the reaction zone width, which is of size $\epsilon$. This means that the thin reaction zone approximation and the concomitant recasting of the problem as a free surface one is uniformly valid at the spiral core.

The above concept is critical to our entire approach and therefore it is worth discussing what other possibilities exist. One way of thinking about the Fife scaling is to recognize that it determines the core size by requiring the relevance of $v$ diffusion. The other possibility is that the core size is determined not 
by $v$ diffusion but instead by the finiteness of the reaction zone; this must occur for example in systems without diffusion of the slow species, but could also occur in general if the selected asymptotic velocity is $\mathscr{C}(1)$ instead of $\mathscr{C}\left(\epsilon^{1 / 3}\right)$. Then, the singular perturbation theory used to derive the governing equation (4) is not valid, and one must return to the coupled PDE system (1), (2) in the inner core region. Of course, whether or not spirals will exist (for some choice of kinetic parameters) in the Fife regime could be studied computationally, but this has not yet been attempted.

\section{Spiral asymptotics}

It is intuitively clear that asymptotically far from the core, the spiral pattern appears to be just a plane wave with some particular asymptotic velocity. In this section, we present a methodology for calculating corrections to the asymptotic shape in a systematic expansion about large $r$. As we shall see, this calculation will place the spiral selection problem in proper focus.

The basic idea is to define new coordinates:

$$
\tilde{\phi}=\phi-\Phi_{0}(r), \quad \tilde{r}=r
$$

This transforms the governing PDE to the form

$$
\left[\left(\frac{\partial}{\partial \tilde{r}}-\frac{\partial \Phi_{0}}{\partial \tilde{r}} \frac{\partial}{\partial \tilde{\phi}}\right)^{2}+\frac{1}{\tilde{r}}\left(\frac{\partial}{\partial \tilde{r}}-\frac{\partial \Phi_{0}}{\partial \tilde{r}} \frac{\partial}{\partial \tilde{\phi}}\right)+\omega \frac{\partial}{\partial \tilde{\phi}}+\frac{1}{\tilde{r}^{2}} \frac{\partial^{2}}{\partial \bar{\phi}^{2}}\right] \tilde{v}_{ \pm} \pm a_{ \pm}=0
$$

The boundary conditions are now imposed at $\tilde{\phi}=0$ and $\tilde{\phi}=2 \pi \lambda_{+} /\left(\lambda_{-}+\lambda_{+}\right)$(for the excited "+" region) and, $-2 \pi \lambda_{-} /\left(\lambda_{-}+\lambda_{+}\right)$(for the quiescent " - "region). Note that one full turn of the spiral pattern corresponds to a change of $\tilde{\phi}$ by $2 \pi$. Note too that $\lambda_{+}$and $\lambda_{-}$, the widths respectively of the "+" and "-" phases, depend on $\tilde{r}$. Using the relationship

$$
c_{\mathrm{n}}=\frac{\omega}{2 \pi}\left[\lambda_{+}(\tilde{r})+\lambda_{-}(\tilde{r})\right]
$$

we can find $\lambda_{-}$given $\lambda_{+}$and the curve $\Phi_{10} ; \lambda_{+}(\tilde{r})$ itself must be found as part of the solution.

To make the above discussion less abstract, let us make use of this formulation to find the asymptotic shape of the spiral. We assume the series

$$
\tilde{v}_{ \pm}(\tilde{r}, \tilde{\phi})=\sum_{n=0}^{\infty} \frac{v_{ \pm}^{(n)}(\tilde{\phi})}{\tilde{r}^{n}}
$$

and

$$
\Phi_{0}(\tilde{r})=-\frac{\omega \bar{r}}{c}+D_{0} \ln \tilde{r}+\sum_{n=1}^{\infty} \frac{D_{n}}{\bar{r}^{n}}
$$


We substitute these expansions into eq. (8), which we then solve order by order in $1 / \tilde{r}$. To leading order

$$
v_{ \pm}^{(0)}=A_{ \pm}^{(0)}+B_{ \pm}^{(0)} \mathrm{e}^{-c^{2} \hat{\phi} / \omega} \pm \frac{a_{ \pm} \tilde{\phi}}{\omega}
$$

Applying the boundary conditions gives six equations

$$
\begin{aligned}
& A_{+}^{(0)}+B_{+}^{(0)}=A_{-}^{(0)}+B_{-}^{(0)}=\nu_{0}^{\prime} c, \\
& B_{+}^{(0)}-B_{-}^{(0)}=-\frac{a_{+}+a_{-}}{c^{2}}, \\
& \tilde{B}_{+}^{(0)}-\tilde{B}_{-}^{(0)}=-\frac{a_{+}+a_{-}}{c^{2}}, \\
& \tilde{B}_{+}^{(0)}+A_{+}^{(0)}-\frac{a_{+}}{c} \lambda_{+}^{(0)}=\tilde{B}_{-}^{(0)}+A_{-}^{(0)}-\frac{a_{-} \lambda_{-}^{(0)}}{c}=-\nu_{0}^{\prime} c .
\end{aligned}
$$

Here $\tilde{B}_{ \pm}^{(0)}=\exp \left( \pm c \lambda_{ \pm}^{(0)}\right) B_{ \pm}^{(0)}$. This is exactly the same system as for plane waves [11, 12] and allows us to determine the unknown coefficients as well as $\lambda_{+}^{(0)}$ and the asymptotic velocity $c$.

Let us now extend this calculation to the first nontrivial correction. The field equation to order $1 / \tilde{r}$ is now inhomogeneous; we find

$$
-\left[\left(\frac{\partial \Phi_{0}}{\partial \tilde{r}}\right)^{2} \frac{\partial^{2}}{\partial \tilde{\phi}^{2}}+\omega \frac{\partial}{\partial \tilde{\phi}}\right] v_{ \pm}^{(1)}=-c B_{ \pm}^{(0)} \mathrm{e}^{-c^{2} \tilde{\phi} / \omega} \pm \frac{a_{ \pm}}{c}-\frac{2 c^{3}}{\omega} D_{0} B_{ \pm}^{(0)} \mathrm{e}^{-c^{2} \tilde{\phi} / \omega}
$$

This is easily solved by

$$
v_{ \pm}^{(1)}=A_{ \pm}^{(1)}+B_{ \pm}^{(1)} \mathrm{e}^{-c^{2} \tilde{\phi} / \omega} \pm \frac{a_{ \pm}}{\omega c} \tilde{\phi}-\frac{c \tilde{\phi}}{\omega} \mathrm{e}^{-c^{2} \tilde{\phi} / \omega} B_{ \pm}^{(0)}\left(1+\frac{2 c^{2}}{\omega} D_{0}\right) .
$$

Again, we have to apply the boundary conditions. To do this, we need the result

$$
c_{\mathrm{n}}=\frac{\omega r}{\sqrt{1+r^{2}\left(\partial \Phi_{0} / \partial r\right)^{2}}} \simeq c+\frac{D_{0} c^{2}}{\omega r}
$$

along with $\kappa=1 / \tilde{r}$ and the assumption $\lambda_{+}(r) /\left[\lambda_{+}(r)+\lambda_{-}(r)\right] \sim \lambda_{+}^{(0)} /\left(\lambda_{+}^{(0)}+\lambda_{-}^{(0)}\right)+E / \tilde{r}$. Applying the boundary conditions gives us, as always, a set of six linear equations in the six unknowns $A_{+}, B_{+}, D_{0}$ and $E$. These can be solved directly.

As a concrete example, we choose the set of parameters derived in ref. [11] from the original Oregonator model; $a_{+}=11 / 16, a_{-}=1 / 16, \gamma=1$ and $\nu_{0}^{\prime}=\sqrt{2} / 30$. We then pick an asymptotic velocity, say $c=0.5$. Solving (13) for the planar interface, we find the results $\lambda_{-}^{(0)}=6.223, \lambda_{+}^{(0)}=0.565$ and the rotation frequency $\omega=2 \pi c /\left(\lambda_{+}^{(0)}+\lambda_{-}^{(0)}\right)=0.463$. We can then evaluate all the lowest-order coefficients and derive the resultant linear system for the first-order shifts; we find

$$
D_{0}=0.2625, \quad E=0 .
$$


Extending this to second order, our result becomes

$$
\begin{aligned}
& \Phi_{0}(r)=-\frac{\omega r}{c}+0.2625 \ln r-\frac{0.194}{r}+\varrho\left(1 / r^{2}\right), \\
& \frac{\lambda_{+}(r)}{\lambda_{+}(r)+\lambda_{-}(r)}=\frac{\lambda_{+}^{(0)}}{\lambda_{+}^{(0)}+\lambda_{-}^{(0)}} .
\end{aligned}
$$

One surprising result of the above calculation is the vanishing of the $E=0$ coefficient to all orders. That is, the relative width $\lambda_{+} / \lambda_{-}$does not vary from its asymptotic value to any order in $1 / \tilde{r}$; this is in distinction to the curve itself, which actually has logarithmically large deviations since $D_{0} \neq 0$. It is possible to make the ansatz $\lambda_{+}(r) / \lambda_{-}(r) \simeq \lambda_{+}^{(0)}(r) / \lambda_{-}^{(0)}(r)+\hat{E} \mathrm{e}^{-\alpha r}$ and derive an eigenvalue equation for $\alpha$. Since this is of only tangential importance, we skip this derivation here.

What is this asymptotic calculation useful for? First, it is obviously possible in principle to measure the asymptotic velocity and compare the above analytic predictions with measured (or computed) shapes. Next, we will see in the next section that this offers a highly non-trivial check on the numerical implementation of the shape equation. Most importantly from our perspective is the statement that these results make regarding the uniqueness of the spiral pattern and in particular of the rotation frequency. Clearly, there is no problem extending this procedure to arbitrarily higher order; we always recover the same structure and can determine the unknown coefficients to each order. Hence, there is no selection of frequency $\omega$. That is, given $\omega$ we can find all other unknowns and construct the expansions (10), (11); we will argue subsequently that this is misleading and in fact the full equation does select $\omega$ albeit by terms that are exponentially small at large distances.

How can we go beyond this asymptotic expansion? At present, the only viable approach appears to be numerical solution of the integro-differential shape equation [11]. The next section discusses the derivation of this equation and the possible form of its solutions.

\section{Shape equation}

In other types of free surface problems [13], it has proven advantageous to recast the equations in the form of an integro-differential shape equation for the actual pattern. To derive this equation we return to the original variables and define

$$
\Psi=v \pm \frac{1}{4} a_{ \pm} r^{2} .
$$

The auxiliary field $\Psi$ then obeys the homogeneous equation $\nabla^{2} \Psi+\omega \partial \Psi / \partial \phi=0$, which furthermore is the same in both phases. Green's theorem then demands that any discontinuities in either $\Psi$ or $\hat{n} \cdot \nabla \Psi$ (for normal to the curve $\hat{n}$ ) give rise to sources in an integral representation of the field. Since $v$ is continuous, the discontinuities of $\Psi$ can be determined directly from eq. (20):

$$
\operatorname{disc} \Psi=\frac{1}{4}\left(a_{+}+a_{-}\right) r^{2}, \quad \operatorname{disc}(\boldsymbol{n} \cdot \nabla \Psi)=\frac{1}{2}\left(a_{+}+a_{-}\right) r \hat{\boldsymbol{n}} \cdot \hat{\boldsymbol{r}} .
$$

Using the above results, we can immediately write down a solution for $\Psi$ in terms of the Green's function

$$
G\left(r, r^{\prime}, \phi, \phi^{\prime}\right)=\int_{-\infty}^{t} \frac{\mathrm{d} t^{\prime}}{4 \pi t^{\prime}} \exp \left\{-\left[r^{2}+r^{\prime 2}-2 r r^{\prime} \cos \left(\phi-\phi^{\prime}-\omega\left(t-t^{\prime}\right)\right)\right] / 4 t^{\prime}\right\} .
$$


Using the easily derived expressions

$$
\operatorname{disc} G=0, \quad \operatorname{disc}\left(\hat{\boldsymbol{n}}^{\prime} \cdot \nabla^{\prime} G\right)=1, \quad \operatorname{disc}\left[(\hat{\boldsymbol{n}} \cdot \nabla)\left(\hat{\boldsymbol{n}}^{\prime} \cdot \nabla^{\prime} G\right)\right]=-c_{\mathrm{n}},
$$

we find

$$
\Psi(r, \phi)=\int \mathrm{d} s^{\prime}\left(\hat{n}^{\prime} \cdot \nabla^{\prime} G\right) \frac{1}{4} r^{\prime 2}\left(a_{+}+a_{-}\right)-\int \mathrm{d} s^{\prime} G\left[\frac{1}{2}\left(\hat{n}^{\prime} \cdot \hat{r}^{\prime}\right) r^{\prime}\left(a_{+}+a_{-}\right)+\frac{1}{4} c_{\mathrm{n}} r^{\prime 2}\left(a_{+}+a_{-}\right)\right]
$$

which determines the field everywhere if we know the actual reaction zone $\Phi_{0}(r)$.

The shape equation now follows when we evaluate $\Psi$ on the reaction zone itself and use the known value of the field $v$ to find an expression for the value of $\Psi$. Doing this, we derive the final shape equation

$$
\frac{\nu_{0}^{\prime}\left(c_{\mathrm{n}}+\gamma \kappa\right)+\frac{1}{4} a_{+} r^{2}}{a_{+}+a_{-}}=-\int \mathrm{d} s^{\prime}\left(\hat{\boldsymbol{n}}^{\prime} \cdot \nabla^{\prime} G\right) \frac{1}{4} r^{\prime 2}+\int \mathrm{d} s^{\prime} G\left[\frac{1}{2}\left(\hat{\boldsymbol{n}}^{\prime} \cdot \hat{\boldsymbol{r}}^{\prime}\right) r^{\prime}+\frac{1}{4} c_{\mathrm{n}} r^{\prime 2}\right]
$$

where the first integral must be evaluated on the " + " side of the interface. By definition, the normal vector $\hat{n}$ always points from "+" to "-". This is then a closed form equation for the curve $\Phi_{0}(r)$ in which the rotation frequency $\omega$ appears as a parameter, both in the Green's function and in the normal velocity $c_{\mathrm{n}}$ given by eq. (16).

In principle, this equation can be solved by discretizing the curve and varying the parameters using some type of Newton's iteration to converge to a final solution. This has not yet been accomplished, but we have performed one specific consistency check. That is, if we take our (lowest order) asymptotic expansion for the spiral shape derived in the last section and evaluate the integral on the right-hand side, it differs from the left-hand side by terms that are no bigger than $\mathscr{O}(1 / r)$. This is rather nontrivial since the generic size of both sides of the equation is $\mathscr{O}\left(r^{2}\right)$.

In the absence of an exact solution, we can employ a heuristic argument that equations of this type should require fixed values for the rotation frequency $\omega$. We can rewrite the shape equation in the schematic form $c_{\mathrm{n}}+\gamma \kappa=F\left[\Phi_{0}(r)\right]$, where $F$ is a rather complex functional of the curve. We know of course that as $s \rightarrow \pm-\infty, F$ will approach $\pm c$, the asymptotic velocity and that furthermore $F$ cannot depend on either absolute angle or absolute arclength. Let us change to treating the curve parametrically by giving the radius $R_{0}$ and the angle $\Phi_{0}$ as functions of the arclength. We can then rewrite the above equation as a dynamical system for the variables $R_{0}(s), \Phi_{0}(s)$ and $\Theta(s) \equiv \cos ^{-1}(\hat{\boldsymbol{n}} \cdot \hat{\mathbf{y}})$, where the $\hat{\boldsymbol{y}}$ coordinate axis has been chosen for a reference direction; after some geometry, this yields [14]

$$
\frac{\mathrm{d} R_{0}}{\mathrm{~d} s}=-\cos \left(\Phi_{0}+\Theta\right), \quad \frac{\mathrm{d} \Phi_{0}}{\mathrm{~d} s}=\frac{\sin \left(\Phi_{0}+\Theta\right)}{R_{0}}, \quad \frac{\mathrm{d} \Theta}{\mathrm{d} s}=\frac{\omega R_{0} \cos \left(\Phi_{0}+\Theta\right)+F}{\gamma} .
$$

Now, we can write down the most general asymptotic spiral solution in terms of four constants $s_{ \pm}, \Phi_{ \pm}$,

$$
\begin{aligned}
& R_{0}(s) \sim \sqrt{(2 c / \omega)\left(s-s_{ \pm}\right)}, \quad s \rightarrow \pm \infty, \quad \Phi_{0}(s) \sim \sqrt{(2 \omega / c)\left(s-s_{ \pm}\right)}+\Phi_{ \pm}, \quad s \rightarrow \pm \infty, \\
& \Theta(s) \sim-\Phi_{0}(s)+3 \pi / 2, \quad s \rightarrow+\infty, \quad \text { and } \quad \Theta(s) \sim-\Phi_{0}(s)+\pi / 2, \quad s \rightarrow-\infty .
\end{aligned}
$$


Of these four unknowns, one corresponds to an overall rotation of the entire system (and is hence irrelevant) and one goes to fix $\lambda_{+} / \lambda_{-}$; we of course have already correctly chosen $c$ and hence $\lambda_{+}+\lambda_{-}$. This leaves two unknowns but three matching conditions to generate a smooth trajectory from $s=-\infty$ to $s=+\infty$. Hence there is generally no solution unless we also pick $\omega$ appropriately. In other words, the frequency is fixed by requiring the matching (at the core) of solutions with the correct behavior at $\pm \infty$ and is not visible in the asymptotic large distance expansion.

The reasoning in the previous paragraph serves to explain why several different "geometrical" models of spirals $[10,14-16]^{\# 3}$ have all yielded unique rotation frequencies. In a geometrical model, $F$ is replaced by a function of local objects and hence eq. (23) can be directly integrated. From our current perspective, any "reasonable" geometrical model will give selection via this general mechanism. This does not really answer the question of whether this actually happens in a physical nonlocal model where there are conceivable loopholes in the above approach; but this does offer one possible scenario as to how the asymptotic freedom of choosing $\omega$ is broken by the core region solution. Eventually, we hope to answer this question more definitely by finding full solutions of eq. (23).

\section{Comments}

To summarize, we have presented a novel way of looking at the spiral selection problem within the Fife scaling limit of the Oregonator model. We have calculated the asymptotic spiral shape, discussed the importance of the diffusion controlled core and reviewed the derivation of a spiral shape equation. Finally, we have presented a general argument as to why the rotation frequency might be selected via matching near the core. An explicit method capable in principle of demonstrating the validity of our proposed scenario was presented, and we are currently attempting to implement this approach.

The last comment we wish to make concerns the tip meandering instability studied in great detail for the $\mathrm{BZ}$ system $[5,17,18]$. Our best guess is that this instability is a remnant of the Hopf bifurcation at zero traverse wavevector known to occur [19] for planar waves as $\lambda_{+}, \lambda_{-} \rightarrow 0$. For the Fife limit, this instability is again tied up with $v$ diffusion and hence will become important on the same general scale as the core. Specifically, as the spiral moves inward from infinity, the width of the wave shrinks, with of course $\lambda_{+} \rightarrow 0$ as we reach the spiral core. Imagine doing a WKB calculation where at each radius we consider the spiral as a locally planar problem with some normal velocity. It appears likely that the stability boundary can be crossed as we move towards the core leading to a locally unstable region. Globally, this would lead to the prediction of an oscillatory mode localized near the core, which is what one appears to observe.

The above conjecture explains why it is possible to see the meandering instability even with $v$ diffusion. Most explanations [20] of the meandering have dealt with simplified models most appropriate to the case of no $v$ diffusion and core sizes of order $\epsilon$. Obviously, a great deal of additional work will be necessary in order to see if this idea makes any sense. We have included this discussion, just to point to that once one can deal with the spiral as a free boundary problem, techniques which have proven capable of finding stability spectra for other free boundary systems [21] can be applied here and may offer additional insight into spiral pattern dynamics.

\footnotetext{
${ }^{\# 3}$ For a review of attempts to do this in the Russian literature, see ref. [15].
} 


\section{References}

[1] R.J. Field and M. Burger, eds., Oscillations and Traveling Waves in Chemical Systems (Wiley, New York, 1985).

[2] S.C. Muller, T. Plesser and B. Hess, Science 230 (1985) 661; Physica D 24 (1987) 87.

[3] A.T. Winfree, When Time Breaks Down (Princeton Univ. Press, Princeton, NJ, 1987).

[4] W.Y. Tam, W. Horsthemke, Z. Noszticzius and H. Swinney, J. Chem. Phys. 88 (1888) 3395.

[5] G.S. Skinner and H. Swinney, Physica D 48 (1991) 1-16.

[6] M.P. Cox, G. Ertl and R. Imbihl, Phys. Rev. Lett. 54 (1985) 1725.

[7] P.C. Newell and F.M. Ross, J. Gen. Microbiol. 128 (1982) 2715.

[8] J.J. Tyson, in: Nonlinear Phenomena in Chemical Dynamics, eds. C. Vidal and A. Pacault (Springer, Berlin, 1981).

[9] P. Fife, in: Nonequilibrium Dynamics in Chemical Systems, eds. C. Vidal and A. Pacault (Springer, Berlin, 1984).

[10] J.P. Keener and J.J. Tyson, Physica D 21 (1986) 307; Physica D 32 (1988) 327; J.P. Keener, SIAM J. Appl. Math. 46 (1986) 1039.

[11] D. Kessler and H. Levine, Physica D 39 (1989) 1.

[12] J.P. Dockery, J.P. Keener and J.J. Tyson, Physica D 30 (1988) 177.

[13] D. Kessler, J. Koplik and H. Levine, Adv. Phys. 37 (1988) 255.

[14] D. Kessler and H. Levine, Europhys. Lett., in press.

[15] J.P. Keener and J.J. Tyson, Physica D 32 (1988) 327.

[16] P. Pelcé and J. Sun, Physica D 48 (1991) 253.

[17] W. Jahnke, W.E. Skaggs and A.T. Winfree, J. Phys. Chem. 93 (1989) 740.

[18] D. Barkeley, M. Kness and L.S. Tuckerman, Phys. Rev. A 42 (1990) 2489.

[19] D. Kessler and H. Levine, Phys. Rev. A 41 (1990) 5148.

[20] A. Mikhailov, unpublished; E. Meron, preprint (1990).

[21] D. Kessler and H. Levine, Phys. Fluids 30 (1987) 1246. 